\title{
Identifying module biomarkers from gastric cancer by differential correlation network
}

This article was published in the following Dove Press journal:

OncoTargets and Therapy

19 September 2016

Number of times this article has been viewed

\author{
Xiaoping Liul ${ }^{1-3, *}$ \\ Xiao Chang ${ }^{1,3, *}$ \\ 'College of Statistics and Applied \\ Mathematics, Anhui University of \\ Finance and Economics, Bengbu, \\ Anhui Province, People's Republic of \\ China; ${ }^{2}$ Key Laboratory of Systems \\ Biology, Shanghai Institutes for \\ Biological Sciences, Chinese Academy \\ of Sciences, Shanghai, People's \\ Republic of China; ${ }^{3}$ Collaborative \\ Research Center for Innovative \\ Mathematical Modeling, Institute of \\ Industrial Science, University of Tokyo, \\ Tokyo, Japan \\ *These authors contributed equally \\ to this work
}

\begin{abstract}
Gastric cancer (stomach cancer) is a severe disease caused by dysregulation of many functionally correlated genes or pathways instead of the mutation of individual genes. Systematic identification of gastric cancer biomarkers can provide insights into the mechanisms underlying this deadly disease and help in the development of new drugs. In this paper, we present a novel network-based approach to predict module biomarkers of gastric cancer that can effectively distinguish the disease from normal samples. Specifically, by assuming that gastric cancer has mainly resulted from dysfunction of biomolecular networks rather than individual genes in an organism, the genes in the module biomarkers are potentially related to gastric cancer. Finally, we identified a module biomarker with 27 genes, and by comparing the module biomarker with known gastric cancer biomarkers, we found that our module biomarker exhibited a greater ability to diagnose the samples with gastric cancer.
\end{abstract}

Keywords: biomarkers, gastric cancer, stomach cancer, differential network

\section{Introduction}

Gastric cancer (stomach cancer), the second most deadly cancer around the world, ${ }^{1}$ is prevalent in East Asia, Eastern Europe, and Central and South America. In the US, about 21,500 cases of gastric cancer were diagnosed in 2008. ${ }^{2}$ However, the mechanism of gastric cancer is not yet clear. Systematic identification of gastric cancer genes can provide insights into the process of tumorigenesis of this deadly disease. Here, cancerrelated genes refer to those genes the dysfunctions or mutations of which are causally implicated in cancer. ${ }^{3}$ However, it is now a well-recognized fact that cancer is not caused by individual genes but by an entire system or network, which involves many correlated genes and dynamic processes. The procedure of identifying cancer-related genes through biological experiments is notoriously difficult and time-consuming. . $^{3,4}$ Nowadays, many computational methods are available to predict cancer-related genes, ${ }^{5,6}$ most of which focus on the differential expression of genes and static regulation relations between genes ${ }^{7,8}$ while ignoring the dynamic regulations or network rewiring between molecules at different disease stages. So a dynamic method that depicts the dynamic variety of regulation between molecules will uncover crucial information that cannot be detected in static conditions. ${ }^{9,10}$ Generally, the regulations or interactions between molecules change dynamically at different times or in different tissues, which are causally related to the disease progression. As the regulations between molecules are changed at different times or in different tissues, these molecules, which interact with different targets on the molecular interaction network, will show a potential correlation with the disease. Therefore, new potential cancer genes can be detected by taking into account the dynamic regulations and network rewiring between molecules,
Correspondence: Xiao Chang

Institute of Statistics and Applied

Mathematics, Anhui University of Finance

and Economics, Bengbu 233030, Anhui,

People's Republic of China

Tel +865523173187

Email chxlaugh@hotmail.com
OncoTargets and Therapy 20I6:9 570I-57II

5701

Dovepress f $y$ in $\mathbf{0}$

http://dx.doi.org/10.21 477/OTT.SI | 13281 (c) (1) 2016 Liu and Chang. This work is published and licensed by Dove Medical Press Limited. The full terms of this license are available at https://www.dovepress.com/terms.php

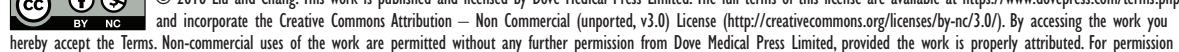
for commercial use of this work, please see paragraphs 4.2 and 5 of our Terms (https://www.dovepress.com/terms.php). 
which may have been ignored when investigating differential expressions only ${ }^{9,10}$ in the previous studies.

In this paper, a novel network-based approach is presented to predict gastric cancer genes by investigating dynamic interactions and network rewiring between molecules. In particular, we assume that the dysfunction or network rewiring of the correlation networks found in people suffering from cancer is possibly related to tumorigenesis or carcinogenesis. Utilizing gene expression to filter genes expressing differently for recognizing rewired networks can improve the power of distinguishing between the normal and cancer samples. As results, we predict 27 gastric cancer genes based on analysis of microarray data of 160 clinic samples. Moreover, results with these 27 genes on three independent validation datasets demonstrate that these genes can be used as effective biomarkers to detect gastric cancers efficiently.

\section{Materials and methods}

\section{Gene expression profiles, correlation coefficient, and differential expression}

The gene expression profiles for gastric cancer were obtained from the Gene Expression Omnibus (GEO) database (ID: GSE27342). It contains 160 samples from 80 cancer tissues and their adjacent noncancerous tissues of 80 nontreated gastric cancer patients. All gene expression profiles were normalized by the robust multiarray averaging (RMA) method and the probe sets were mapped to the corresponding gene symbols, omitting the nonmapped probe sets. The expression value of a gene symbol was calculated by averaging the expression values of probe sets of this gene symbol. The clinic information for the stage status of samples. The information about stage of gastric cancer was obtained from the original paper of the dataset. ${ }^{11}$ Since there were fewer samples in Stage I and II than the other stages, the four stages of expression profiles were combined into three phases (Table 1). So, we considered that there were eleven samples in the early phase of gastric cancer from the combination of Stage I and II, 54 samples in the middle phase of gastric

Table I Stages and phases of I 60 clinical samples

\begin{tabular}{lll}
\hline Characteristics & Number of samples & Phases \\
\hline Stages $(\mathrm{n}=80)$ & & \\
I & 4 & Early phase \\
II & 7 & \\
III & 54 & Middle phase \\
IV & 15 & Late phase \\
\hline
\end{tabular}

Note: $\mathrm{n}$ is the number of samples in each group. cancer from stage III, and 15 samples in the late phase of gastric cancer from stage IV (Table 1). The information for the patients was also included in Table 1 . The human network was constructed by calculating the Pearson correlation coefficient (PCC) between any two genes in the dataset; each $P$-value of PCC was corrected by the false discovery rate (FDR) and the gene pairs with corrected $P$-value of PCC $<0.05$ were chosen as the edges of the correlation network. In contrast, if the corrected $P$-value of PCC for the gene pairs was $>0.05$, the edges for the gene pairs were ignored in the correlation network. The differentially expressed genes were tested by the $P$-value of Student's $t$-test between normal and cancer samples. If the $P$-value of a gene for Student's $t$-test was $<0.05$, then the gene would be considered as a differential expressed gene between normal and cancer status. If the $P$-value of a gene for the Student's $t$-test is more than 0.05 , then the gene would be considered as a non-differential expressed gene between normal and cancer status.

For calculating the PCC and Student's $t$-test, a free package named "scipy" (https://www.scipy.org/) for python programming language (https://www.python.org/) was employed, and it calculated the $P$-value of PCC and $t$-test. The FDR correction for $P$-value was done by the "stats" package in $\mathrm{R}$ programming language. ${ }^{12}$

\section{Differential network and candidate biomarker genes}

The gene expression profiles were divided into three phases based on the stages of gastric cancer, as shown in Table 1; in every phase, the gene expression profiles were divided into two groups of normal and cancer, and each group only contained normal or cancer samples in this phase. The correlation coefficient of each interaction within the correlation network was calculated based on the samples of every group. The highly correlated edges were reserved, and lowly correlated edges were deleted. The highly correlated edges were able to identify the interactions among nodes at the corresponding phenotypes. The correlation network was rewired based on these highly correlated edges from the normal and cancer samples individually. The new correlation networks were called network in normal and network in cancer, and are shown in Figure 1. The correlation coefficient threshold value was set to FDR corrected $P$-value $\leq 0.05$, and was used to divide the highly correlated edges and lowly correlated edges. The context-specific network will only keep the highly correlated edges in the network. The differential network between normal and cancer samples could show the dynamic changes of interactions between the two samples. 


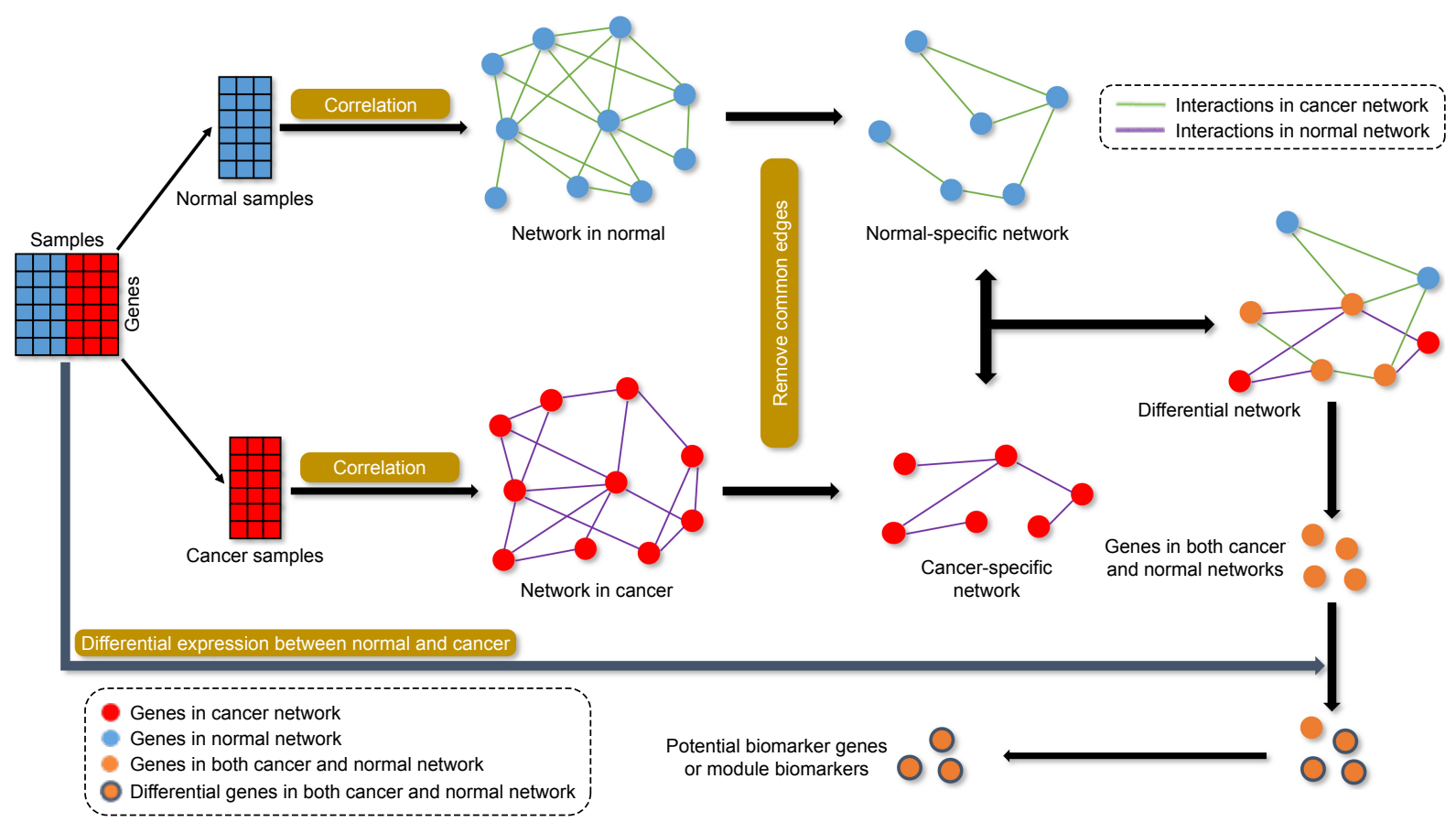

Figure I Schematic flowchart of identifying potential genes based on differential correlation network and gene expression for each phase.

Notes: The threshold for high correlation was set to FDR corrected P-value of 0.05 . "Remove common edges" means removing the interactions that appear in both normal and cancer network, and "Differential expression between normal and cancer" means identifying the differentially expressed genes between normal and cancer with $P$-value 0.05 by Student's $t$-test.

Abbreviation: FDR, false discovery rate.

The normal-specific and cancer-specific networks were individually built by removing the common edges of correlation network in normal and cancer status. The differential networks were formed to combine the normal-specific and cancer-specific networks (Figure 1).

Every edge in the differential network represents the dynamic interaction between the normal and cancer samples, and the edges in the differential network only appear at one phenotype of normal or cancer samples. The disease-related genes were the common genes in both the normal-specific and cancer-specific networks. These genes appear in both normal-specific and cancer-specific network. It is highly possible that these genes play crucial roles in the transformation from normal to cancer status. Then, the differentially expressed genes were detected by $P$-value of 0.05 obtained by Student's $t$-test, and the genes in the differential network were filtered by the differential expressed genes to obtain the potential biomarker genes or module biomarkers for each phase (Figure 1). The common members of the intersection genes at the three phases of gastric cancer were obtained and regarded as candidate biomarker genes or module biomarkers (Figure 2). The candidate biomarker genes or module biomarkers stably appear in the differential network of every phase and always connect with both normal-specific and cancer-specific networks, and these indicate their important dysfunctions from normal to cancer status.

\section{Candidate biomarker genes as module biomarker}

The expression profiles of candidate biomarker genes were used to test the classification ability for normal and cancer phenotypes. We used a support vector machine (SVM) regression method to implement the classification. Given a set of training gene expression samples, each categorized as normal or cancer, SVM classifier builds a model to predict whether a new sample falls into normal or gastric cancer phenotypes. Receiver operating characteristic (ROC) curve and area under the curve (AUC) were used to show the classification efficiency. Furthermore, the two independent datasets of the human gastric tumor were also utilized to check the results of classification in these candidate biomarker genes. Two R ${ }^{12}$ packages "kernlab" and "ROCR" were used to implement the SVM classifier and produce the ROC curve with default parameters. Other three microarray datasets (GSE19826, GSE13911, and GSE2701) and RNA-Seq datasets (The Cancer Genome Atlas [TCGA]) for gastric cancer were chosen for validating the ability of classification for the module biomarkers to distinguish the gastric cancer sample. 


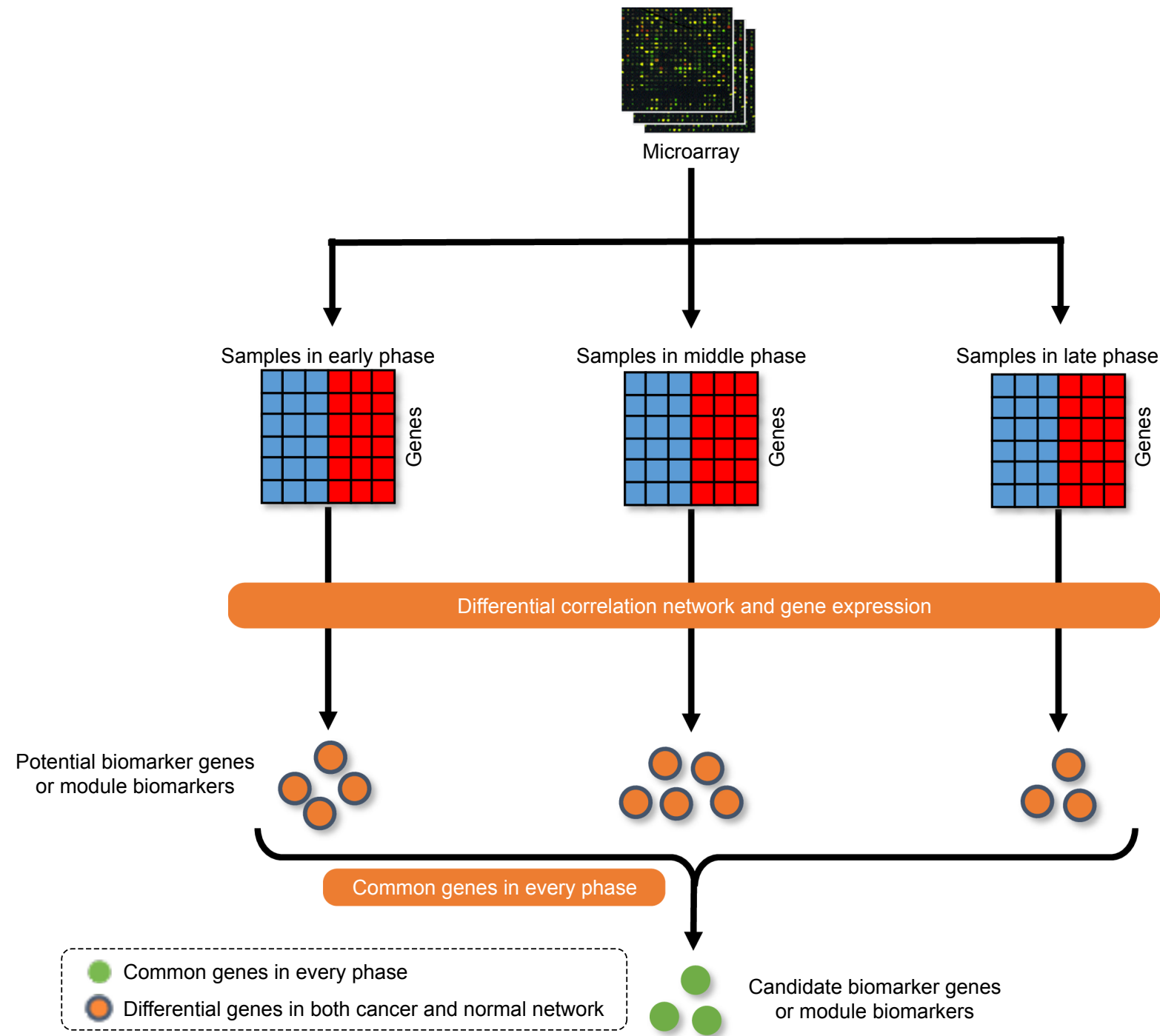

Figure 2 Schematic flowchart of identifying candidate biomarker genes or module biomarkers for gastric cancer.

Note: The overlapped genes of the potential biomarker genes or module biomarkers in the three phases were the candidate biomarker genes or module biomarkers for the method.

\section{Results}

\section{Identification of differential correlation network and gastric cancer genes}

Figure 1 shows the flowchart of our method. All samples were separated into three phases according to their descriptions (Table 1). The normal- and cancer-specific correlation networks were constructed in each phase based on the gene expression data and human correlation network (the details can be found in Materials and methods), respectively, where the common edges between the normal and cancer networks were removed. The differential network was obtained by merging the normal-specific and cancer-specific networks in the corresponding phase, which included the edges that occurred only in normal- or cancer-specific networks. As a result, only those genes that have edges in the differential network and are differentially expressed in both normal and cancer samples are regarded as potential gastric cancer genes in every phase. Furthermore, the differentially expressed genes between normal and cancer status were used to filter the genes in the differential network, because the differentially expressed genes showed greater ability for distinguishing the phenotype. Finally, the genes that occurred in all three phases were treated as our final predicted gastric cancer biomarker genes. In this study, 27 genes were predicted as gastric cancer biomarker genes. These 27 gastric cancer biomarker genes appear in every phase of gastric cancer but interact with different partners (Figure 3). The simple functional analysis of these biomarker genes in PANTHER database was shown to be associated with the cellular, 
metabolic, and developmental processes (Figure 4). Furthermore, the function annotations were conducted for the candidate biomarker genes by further analysis of DAVID database $^{13}$ (Table 2). From gene functions, we could see that some of the candidate biomarker genes had been reported to play an important role in tumorigenesis. For example, $M C M 3$, which encodes one of mini-chromosome maintenance proteins and is involved in the initiation

A
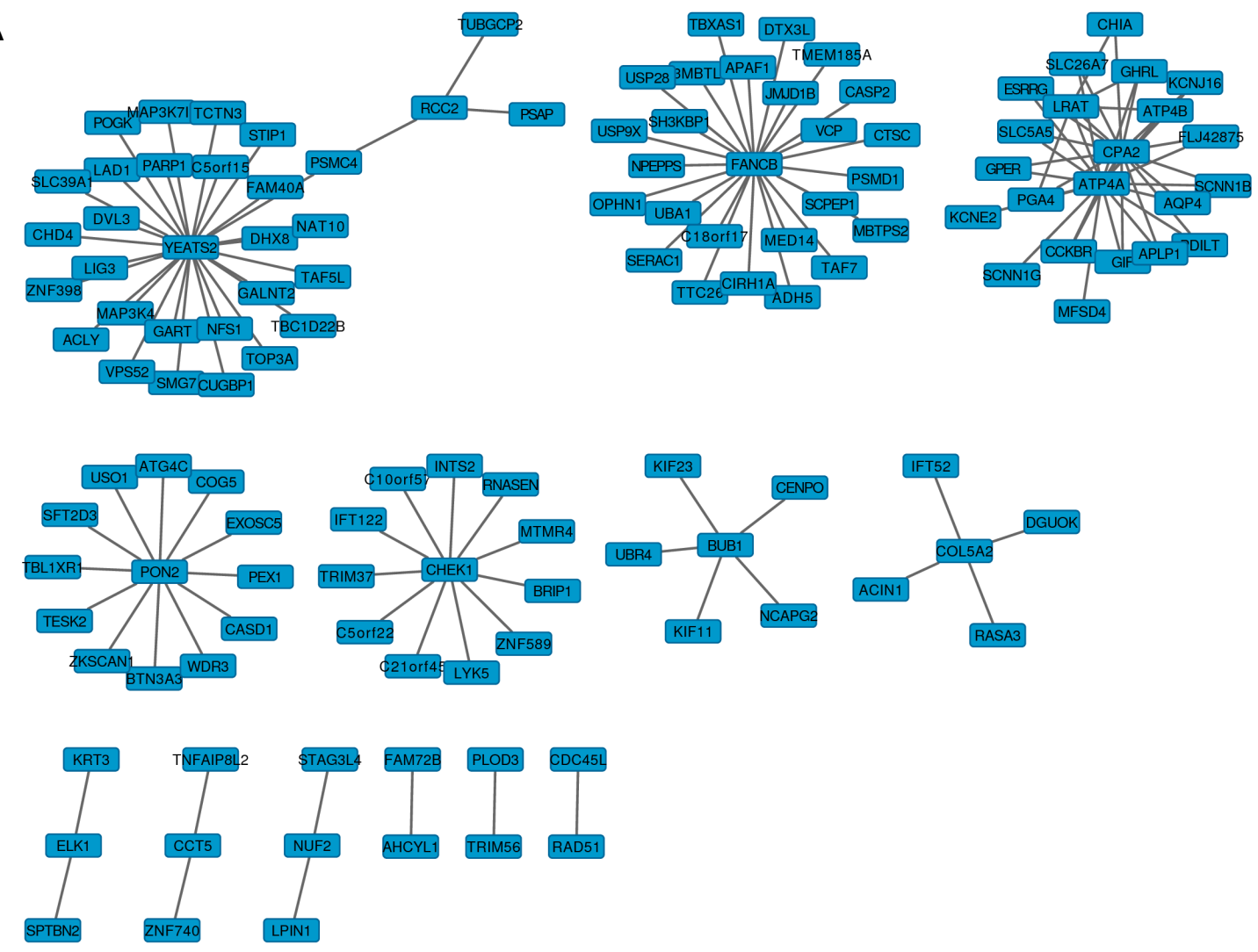

B
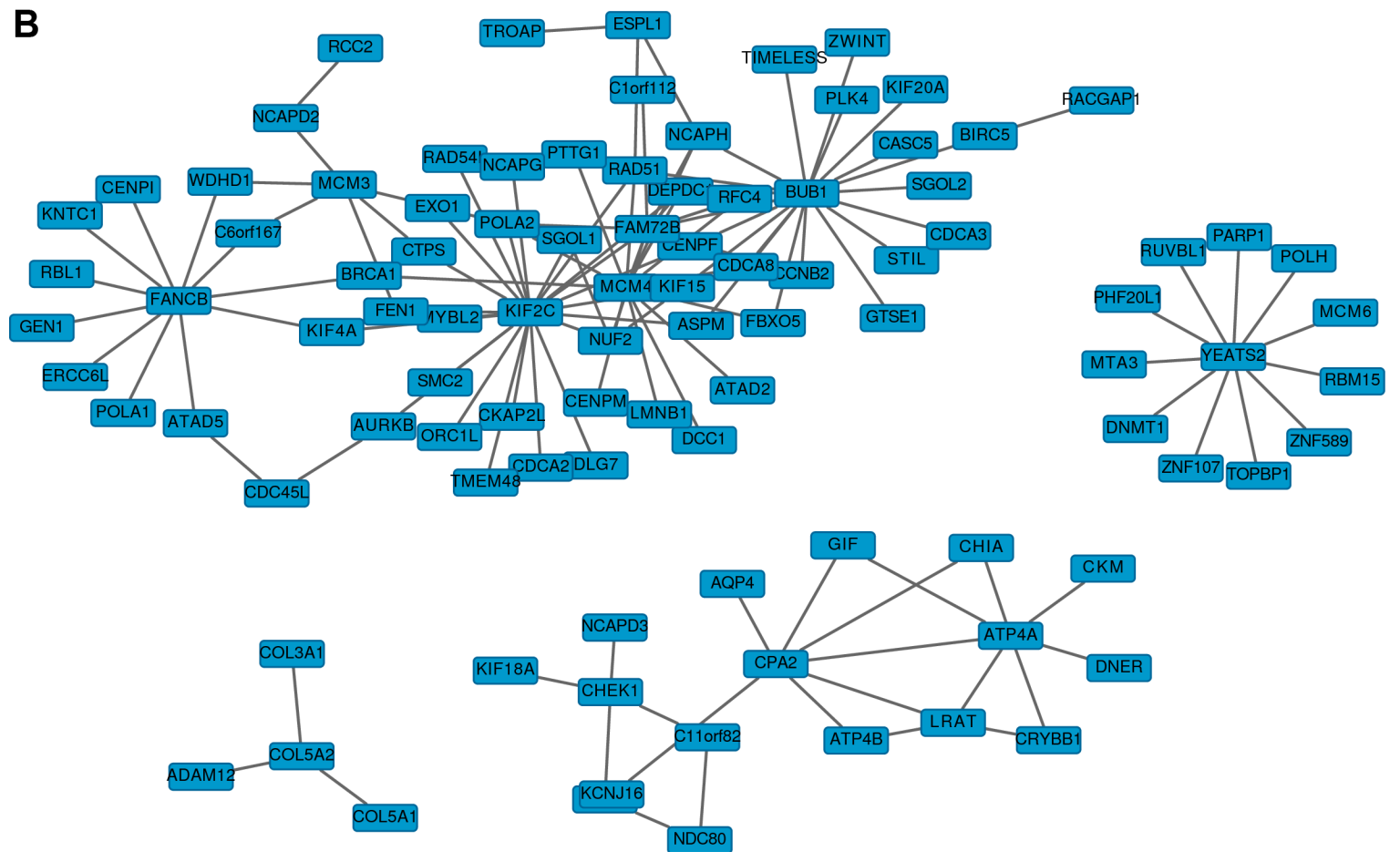

Figure 3 (Continued) 


\section{C}

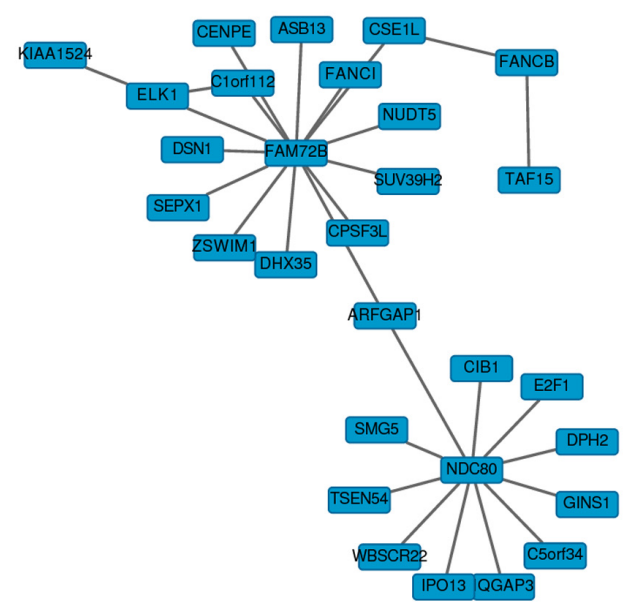

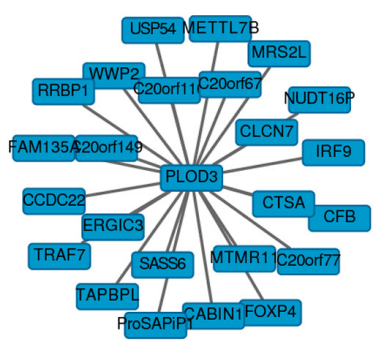
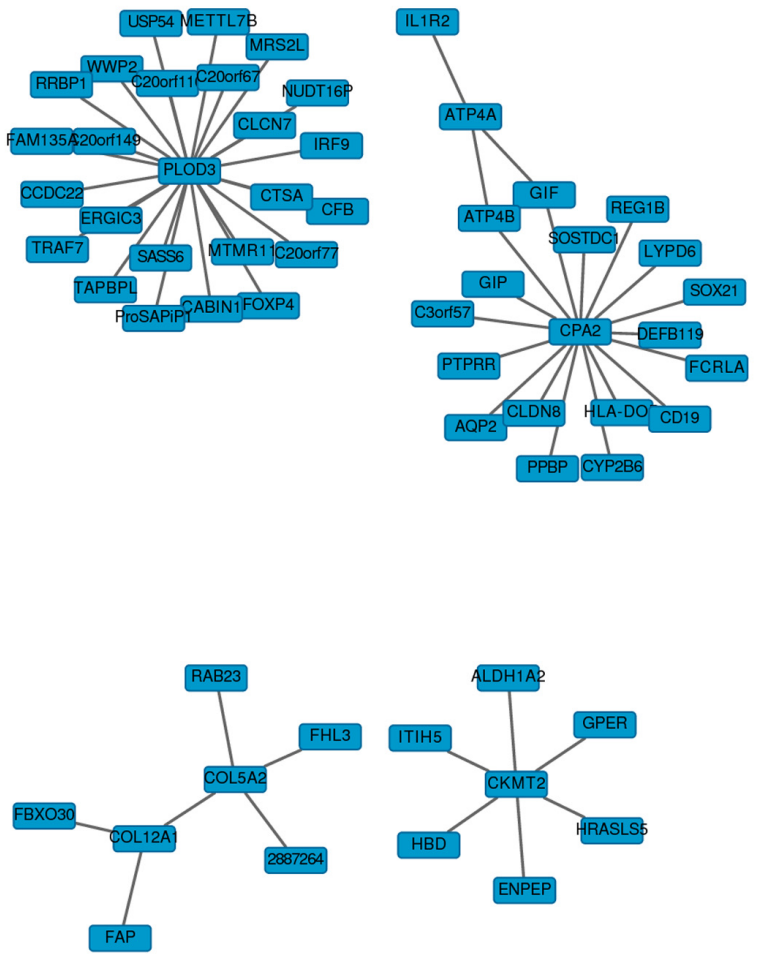

PBK B B B 1
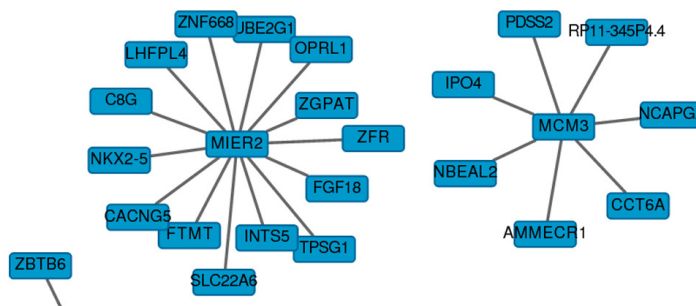

$\int_{\text {MCM4 }}^{2 B T B 6}$<smiles>CCCCC</smiles>

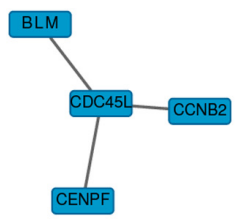<smiles>CC(C)(C)C</smiles><smiles>[10BH][10BH][10BH]</smiles><smiles>CNC</smiles><smiles>C[10BH]C</smiles>

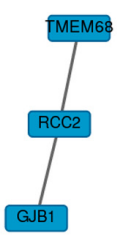

CENPH-YEATS2

CDRT4-PON2

140r1104-FBXL13

\section{D}

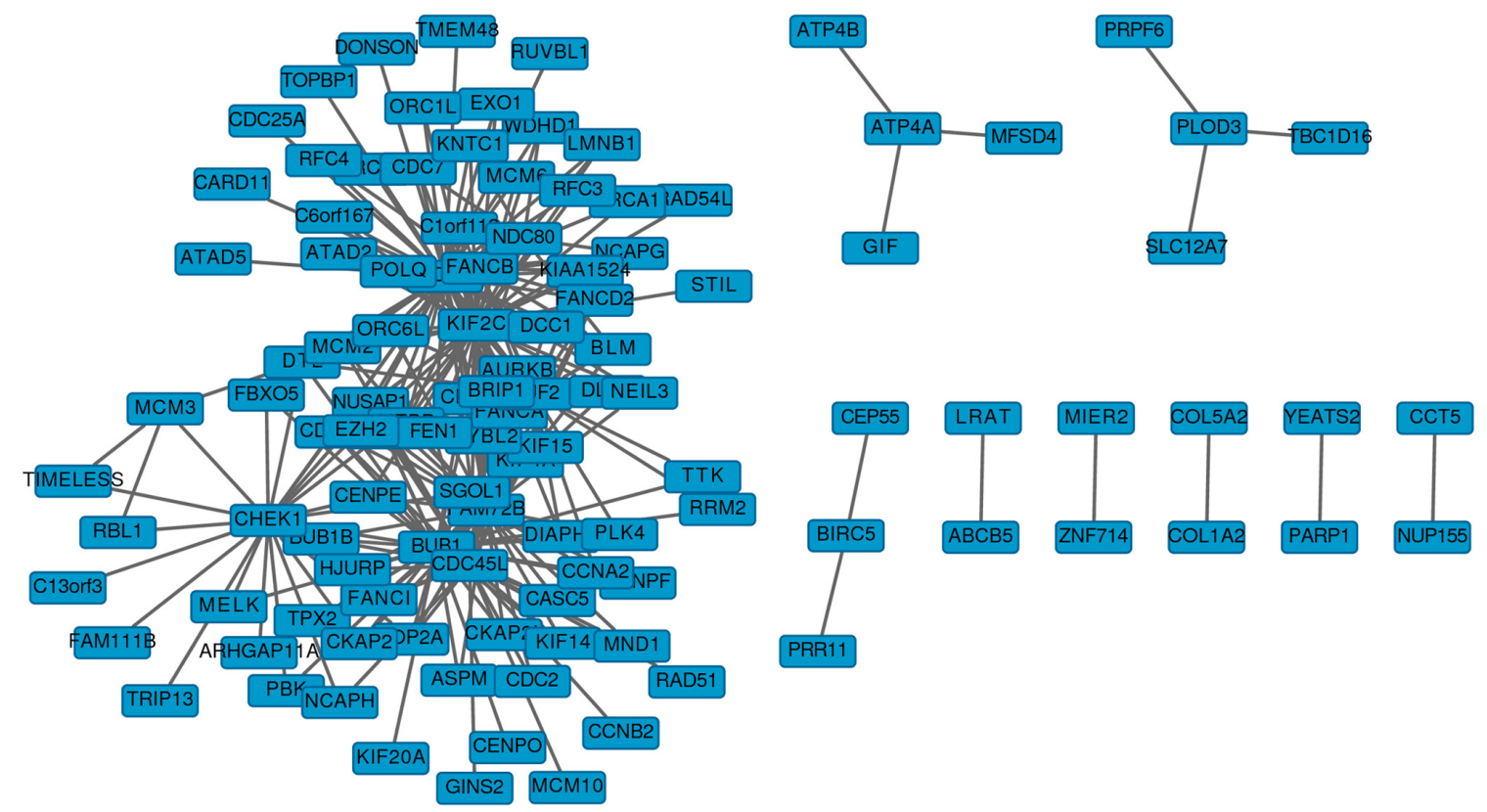

Figure 3 The correlation network involving our predicted module biomarkers and their corresponding interaction partners (first neighbors) in different cancer phases.

Notes: (A) Normal specific network, (B) early phase-specific network, (C) middle phase-specific network, and (D) late phase-specific network. 


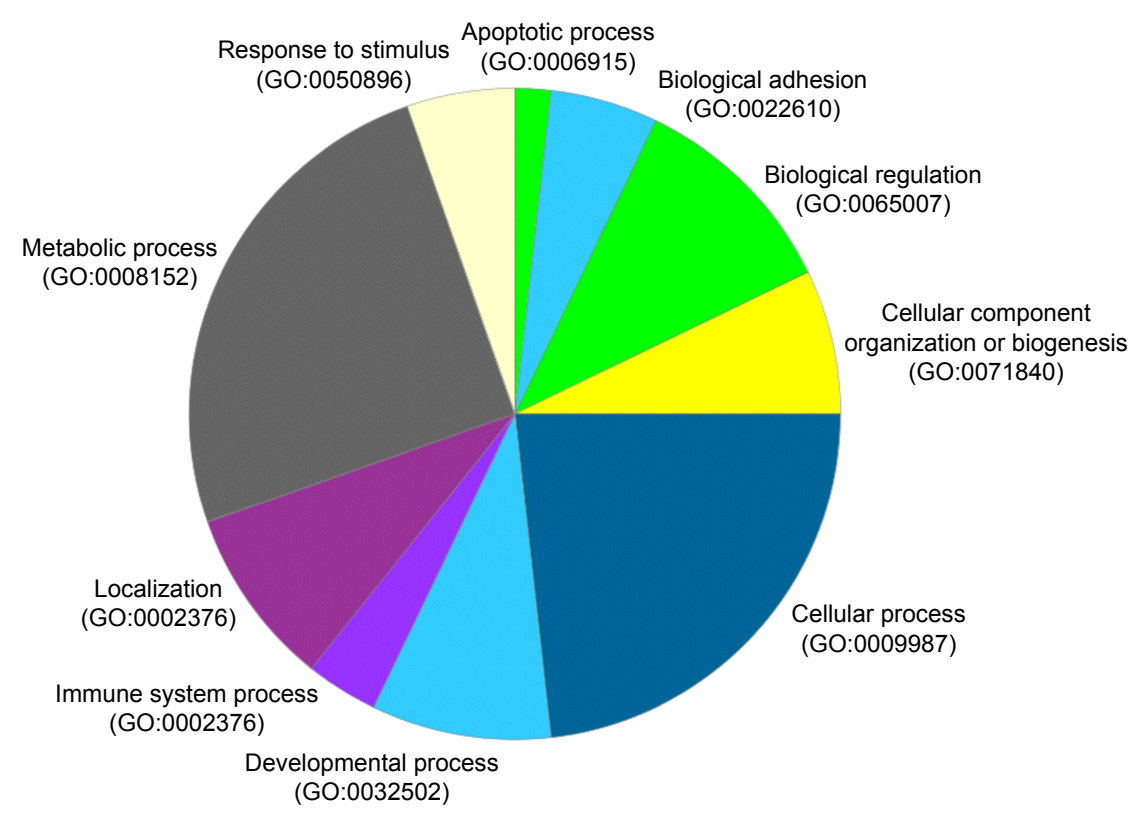

Figure 4 Pie chart of the functional enrichment distribution for the module biomarkers.

Note: This figure showed the distribution of functional enrichment for the module biomarkers based on the biological process of gene ontology analysis.

Table 2 Gene symbols and functional annotations of candidate cancer genes

\begin{tabular}{ll}
\hline Gene symbols & Function annotation in publication \\
\hline FAM72B & Family with sequence similarity 72 member B \\
MCM4 & Mini-chromosome maintenance complex \\
& component 4 \\
PLOD3 & Procollagen-lysine, 2-oxoglutarate 5-dioxygenase 3 \\
CDC45L & Cell division cycle 45 \\
ATP4A & ATPase H+/K+ transporting alpha subunit \\
CHEKI & Checkpoint kinase I \\
CPA2 & Carboxypeptidase A2 \\
RCC2 & Regulator of chromosome condensation 2 \\
MIER2 & MIER family member 2 \\
LRAT & Lecithin retinol acyltransferase \\
& (phosphatidylcholine-retinol O-acyltransferase) \\
YEATS2 & YEATS domain containing 2 \\
MCM3 & Mini-chromosome maintenance complex \\
& component 3 \\
KIF2C & Kinesin family member 2C \\
COL5A2 & Collagen type V alpha 2 \\
FBXLI3 & F-box and leucine-rich repeat protein I3 \\
NUF2 & NUF2, NDC80 kinetochore complex component \\
ESPLI & Extra spindle pole bodies like I, separase \\
BIRC5 & Baculoviral IAP repeat containing 5 \\
CKMT2 & Creatine kinase, mitochondrial 2 \\
ELKI & ELKI, member of ETS oncogene family \\
NDC80 & NDC80 kinetochore complex component \\
CCT5 & Chaperonin containing TCPI subunit 5 \\
COLI2AI & Collagen type XII alpha I \\
FANCB & Fanconi anemia complementation group B \\
PON2 & Paraoxonase 2 \\
JTVI & Aminoacyl tRNA synthetase complex-interacting \\
BUBI & multifunctional protein 2 \\
\hline BOBI mitotic checkpoint serine/threonine kinase \\
\hline
\end{tabular}

Note: The basic functional annotations of the 27 candidate biomarker genes were from the NCBI database.

Abbreviations: ETS, E26 transformation-specific; NCBI, National Center for Biotechnology Information. of genome replication, is involved in gastric, lung, and kidney cancers, ${ }^{14}$ and CHK1, which encodes a Ser/Thr protein kinase and works for checkpoint-mediated cell cycle arrest, is involved in breast ${ }^{15}$ and ovarian cancers. ${ }^{16}$ The functional enrichments of the module biomarkers were involved in mitosis and cell cycle phase (Table 3) according to gene ontology analysis in two web services Gene Ontology Consortium (http://geneontology.org/ page/go-enrichment-analysis) and g:Profiler (http://biit. cs.ut.ee/gprofiler/), and the major functions in which the module biomarkers were involved were associated with cancer processes.

In addition, the network that contained the candidate marker genes and their interaction partners built in each phase was constructed (Figure 3). Network ontology analysis (NOA), ${ }^{17}$ which annotates biological networks, was used to analyze the enriched functions of the network in every phase. The results of NOA are shown in Table 4 . In the normal status, the functions are enriched in signaling pathway, while in the early phase, the functions are enriched in nucleic acid-related metabolic process. The functions of the network change to nuclear division and response to stimulus in the middle and late phase, respectively. The functional annotations of the network in each phase demonstrate clearly that the identified cancer genes function reasonably and their changes reflect the dynamic transition between different stages and transcription, which is an important marker of a cancer cell. 
Table 3 Functional enrichment of the module biomarkers by gene ontology

\begin{tabular}{|c|c|c|c|c|c|}
\hline \multicolumn{3}{|c|}{ Gene ontology consortium } & \multicolumn{3}{|l|}{ g:Profiler } \\
\hline GO item & Functions & $P$-value & GO item & Functions & $P$-value \\
\hline GO:0098763 & Mitotic cell cycle phase & $2.99 \times 10^{-7}$ & GO:0007067 & Mitotic nuclear division & $8.85 \times 10^{-5}$ \\
\hline GO:0022403 & Cell cycle phase & $3.17 \times 10^{-7}$ & GO:0007059 & Chromosome segregation & $9.90 \times 10^{-5}$ \\
\hline GO:005I322 & Anaphase & $5.25 \times 10^{-6}$ & GO:1903047 & Mitotic cell cycle process & $1.18 \times 10^{-4}$ \\
\hline GO:0000236 & Mitotic prometaphase & $1.48 \times 10^{-5}$ & GO:0000280 & Nuclear division & $6.17 \times 10^{-4}$ \\
\hline GO:0000087 & Mitotic M phase & $4.81 \times 10^{-5}$ & GO:005I30I & Cell division & $2.80 \times 10^{-3}$ \\
\hline
\end{tabular}

Note: Top five function enrichment in gene ontology.

\section{Validation of candidate gastric cancer biomarker genes}

In order to further validate the predicted gastric cancer genes, we applied these genes to separate cancer samples from normal samples. Three independent gene expression (microarray) datasets (GSE19826, GSE2701, and GSE13911) of gastric cancer were obtained from GEO database (http://www.ncbi. nlm.nih.gov/geo/) to validate the ability of classification of the module biomarker. At the same time, an RNA-Seq dataset of gastric cancer with 33 normal samples and 183 tumor samples was downloaded from TCGA database (http://cancergenome. nih.gov) to validate the ability of classification for the module biomarker on the differential data type. If these genes can successfully separate cancer samples from control samples for gastric cancer, we predicted that these genes can be proven to be related to gastric cancer. We used the five-fold cross-validation for SVM to detect the ability of classification for our predicted module biomarkers, and the AUC of ROC curve can be used to evaluate the ability of classification for biomarkers, so we can see that the AUCs of ROC curves for three of the four gastric cancer datasets are $>0.9$ and only one AUC is 0.88 for one dataset (Figure 5), and the AUC of ROC curves for the RNA-Seq data was also $>0.9$ (Figure 6). It means that our module biomarkers have a strong ability to identify gastric cancer and that these biomarker genes are strongly related to gastric cancer.

For avoiding the bias of validation of the module biomarker, we compared our results with those obtained with published biomarker sets for gastric cancer, ${ }^{11}$ derived from the differential expression; a five-fold cross-validation was used to evaluate the performance based on different biomarkers, while SVM was used as a classifier. The ability of classification for the two biomarkers to the four microarray datasets and one RNA-Seq was performed and the ROC curves and AUCs are shown in Figures 5 and 6 . The AUC is 0.888 for the module biomarkers and 0.8648 for the known biomarker in Figure 5A, 0.9703 for the module biomarkers and 0.9567 for the known biomarkers in Figure 5B, and 0.9935 for module biomarkers and 0.9748 for known biomarkers in Figure 6; they showed very similar results for the two different biomarkers in Figures 5A, B and 6. It means that the module biomarkers and known public biomarkers for this dataset, GSE13911 and RNA-Seq datasets, have similar ability of classification. The AUC is 0.9933 for the module biomarkers and 0.8969 for the known biomarker in Figure 5C, and 0.9222 for the module biomarkers and 0.7889 for the known biomarkers in Figure 5D. Greater AUCs were obtained for module biomarkers than known biomarkers, as shown in Figure 5C and D, which means that module biomarkers showed better effects in the datasets GSE2701 and GSE19826. So the ability of classification of module biomarkers identified by our study is not worse than the known biomarkers for gastric cancer. In most cases, the ability of classification of the module biomarkers was better than the known biomarkers from differential expression test for the gastric cancer dataset.

Table 4 NOA of networks involving candidate biomarker genes

\begin{tabular}{llll}
\hline Normal & Early phase & Middle phase & Late phase \\
\hline Cell cycle & Heterocycle metabolic process & Nuclear division & lon transport \\
(GO:0007049) & (GO:0046483) & (GO:0000280) & (GO:00068II) \\
Regulation of cellular process & Spindle organization & Organelle fission & Monovalent inorganic cation transport \\
(GO:0050794) & (GO:000705I) & (GO:0048285) & (GO:00I5672) \\
DNA replication & Small molecule metabolic process & Mitosis & Response to stimulus \\
(GO:0006260) & (GO:004428I) & (GO:0007067) & (GO:0050896) \\
\hline
\end{tabular}

Notes: The table shows the result of NOA for networks composed by the candidate biomarker genes and their directly connected genes. We can see that the network functions change from early to late phase of cancer. The three top significant results from NOA are listed in the table.

Abbreviation: NOA, network ontology analysis. 

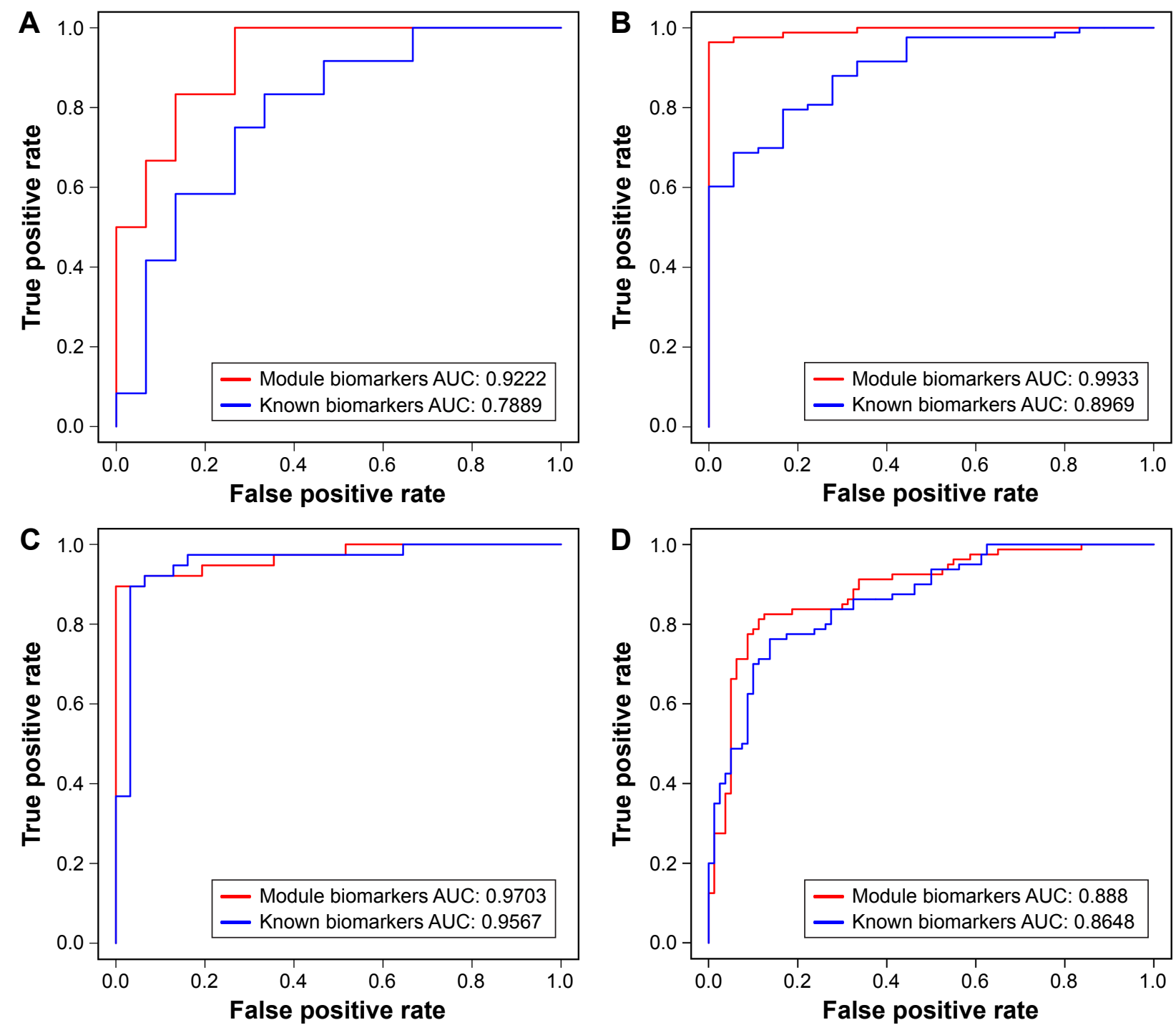

Figure 5 ROC curves obtained with our module markers and published biomarkers. Notes: (A) The gene expression dataset used to identify differential network, and three independent datasets GSEI39II (B), GSE270I (C), and GSEI9826 (D) that were not used in this work.

Abbreviations: AUC, area under the curve; ROC, receiver operating characteristic.

\section{Discussion}

In this paper, we have proposed a new method to detect potential module biomarkers from a variety of network topology and gene expression between normal and cancer status for gastric cancer. There is a module biomarker with 27 genes identified in this study, and this module biomarker showed a powerful ability to classify the phenotypes between normal and cancer status. In this method, the key genes from a variety of network topology can be detected by differential correlation network, and then they can be further filtered by the differentially expressed genes which are not the significant differential expressed between normal and cancer status. So the ability of classification of the module biomarker would be better than the traditional markers, which only come from differentially expressed genes. Although there are only 27 genes in the module biomarker, many genes in the module biomarker are important factors in the development and diagnosis of gastric cancer. For example, ATP4A, which encodes a proton pump that catalyzes the hydrolysis of ATP coupled with the exchange of hydrogen and potassium ions across the plasma membrane, is an important serum biomarker for gastric cancer $^{18}$ and plays a critical role in gastric neuroendocrine tumor. ${ }^{19} \mathrm{RCC} 2$ is cell cycle gene regulator and can promote directional cell migration, ${ }^{20,21}$ but the aberrant expression of RCC 2 can confer a growth advantage on tumor cells in gastric cancer. ${ }^{22} \mathrm{KIF} 2 \mathrm{C}$, which encodes a microtubule-dependent molecular motor and can depolymerize microtubules at the plus end, is overexpressed in human gastric cancer and 


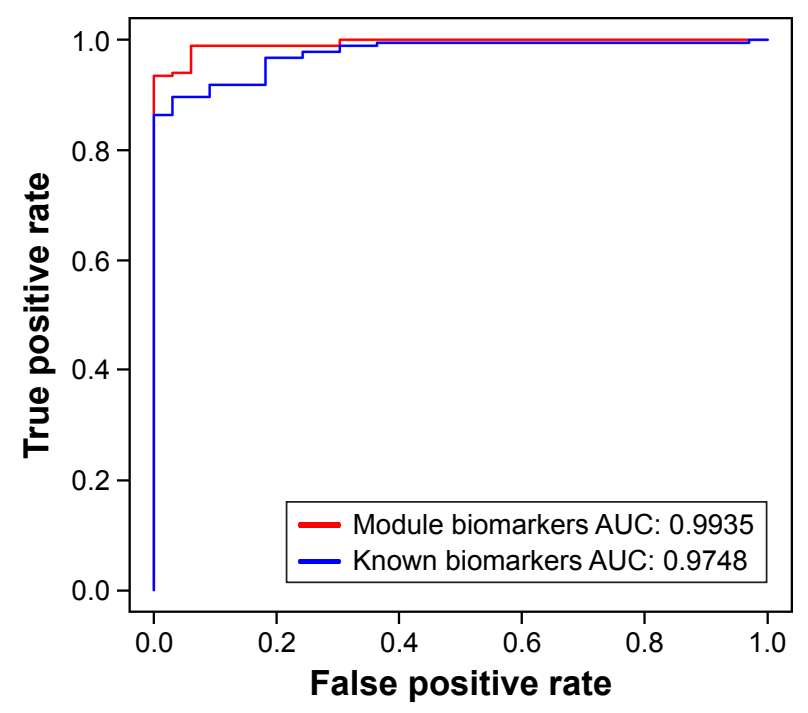

Figure 6 ROC curves for RNA-Seq data from TCGA.

Notes: This figure shows the ROC curves for the classification of our module markers and published biomarkers based on RNA-Seq data of gastric cancer from TCGA.

Abbreviations: AUC, area under the curve; ROC, receiver operating characteristic; TCGA, The Cancer Genome Atlas.

associated with lymphatic invasion and lymph node metastasis in gastric cancer. ${ }^{23}$ BUB1 is a mitotic checkpoint gene and plays an important role at the spindle assembly checkpoint; its overexpression is correlated with tumor proliferating activity in human gastric cancer. ${ }^{24,25}$ Most of the other genes in the module biomarker have also been reported to play critical roles in other tumors or cancers. For example, the MCM4, which is one of the mini-chromosome maintenance proteins, is also an important factor in the tumor process and can be a biomarker in non-small-cell lung cancer. ${ }^{26,27} \mathrm{CDC} 45 \mathrm{~L}$, which is an essential protein required for the initiation of DNA replication, is an important factor in lung cancer and a potentially useful target for lung cancer immunotherapy. ${ }^{28}$ MCM3 and CHEK1 are involved in cancer processes. ${ }^{14-16}$

The $P$-value of Pearson correlation coefficient was used as the threshold for high correlation, because there is a different number of samples in the three phases (eleven samples in early phase, 54 samples in middle phase, and 15 samples in late phase) of gastric cancer.

All the genes in the module biomarker are differentially expressed genes in both normal and cancer samples, so they can be used as biomarkers to distinguish the gastric cancer samples from normal samples. As these genes fall into the same module in the network, they have a better combination effect on the classification of phenotypes than only differentially expressed genes. The results of classification show that the ability of classification of the module biomarker is similar or better than the known biomarkers from only differentially expressed genes, based on microarray data from GEO and the RNA-Seq data from TCGA.

In this paper, we obtained a module biomarker with 27 genes. The 27 genes were from common potential biomarker genes of three phases of gastric cancer (Figure 2), so there are no stage- or phase-specific biomarker genes in the 27 genes. Of course, the method can also be used to obtain some stage- or phase-specific biomarker genes for gastric cancer.

\section{Conclusion}

In this paper, we have proposed a new network-based method to detect the module biomarkers for complex disease, and a module biomarker with 27 candidate genes was identified for gastric cancer. The module biomarker of gastric cancer can help to diagnose and treat this deadly cancer efficiently, and also shed light on other cancers. Some genes in the module biomarkers are known cancer genes and have been reported in the literature, eg, ATP4A, MCM3, and CHEK1.

We validated the ability of classification of this module biomarker by SVM and the results of AUC showed a great ability of this module biomarker to distinguish the cancer samples and normal samples in gastric cancer. By comparing the ability of classification of known biomarkers from differential expression for gastric cancer, the module biomarker showed better results for classification of gastric cancer data, and the results showed that the module biomarkers are more powerful than the known biomarkers in classifying the gastric samples.

\section{Acknowledgments}

This work is partly supported by National Natural Science Foundation of China (Grant No: 61403363), China Postdoctoral Science Foundation (Grant No: 2014T70441 and 2013M541565), and Key Project of Natural Science of Anhui Provincial Education Department (Grant No: KJ2016A002).

\section{Disclosure}

The authors report no conflicts of interest in this work.

\section{References}

1. Parkin DM, Bray F, Ferlay J, Pisani P. Global cancer statistics, 2002. CA Cancer J Clin. 2005;55(2):74-108.

2. Parkin DM, Bray F, Ferlay J, Pisani P. Gastric cancer. Br Med Bull. 2008;85:84-100.

3. Futreal PA, Coin L, Marshall M, et al. A census of human cancer genes. Nat Rev Cancer. 2004;4(3):177-183.

4. Polager S, Ginsberg D. p53 and E2f: partners in life and death. Nat Rev Cancer. 2009;9(10):738-748. 
5. Chuang HY, Lee E, Liu YT, Lee D, Ideker T. Network-based classification of breast cancer metastasis. Mol Syst Biol. 2007;3:140.

6. Liu ZP, Wang Y, Zhang XS, Xia W, Chen L. Detecting and analyzing differentially activated pathways in brain regions of Alzheimer's disease patients. Mol Biosyst. 2011;7(5):1442-1452.

7. Liu X, Tang WH, Zhao XM, Chen L. A network approach to predict pathogenic genes for Fusarium graminearum. PLoS One. 2010;5(10): e13021.

8. Chen L, Wang RS, Zhang XS. Biomolecular Networks. Hoboken, New Jersey: John Wiley \& Sons, Inc; 2009.

9. Bandyopadhyay S, Mehta M, Kuo D, et al. Rewiring of genetic networks in response to DNA damage. Science. 2010;330(6009):1385-1389.

10. Liu X, Liu ZP, Zhao XM, Chen L. Identifying disease genes and module biomarkers by differential interactions. J Am Med Inform Assoc. 2012; 19(2):241-248.

11. Cui J, Chen Y, Chou WC, et al. An integrated transcriptomic and computational analysis for biomarker identification in gastric cancer. Nucleic Acids Res. 2011;39(4):1197-1207.

12. R Development Core Team. R: a language and environment for statistical computing. R Foundation for statistical computing. Vienna, Austria; 2011. Available from: http://www.R-project.org/. ISBN 3-900051-07-0.

13. Huang DW, Sherman BT, Lempicki RA. Systematic and integrative analysis of large gene lists using DAVID bioinformatics resources Nat Protoc. 2009;4:44-57.

14. Ha SA, Shin SM, Namkoong H, et al. Cancer-associated expression of minichromosome maintenance 3 gene in several human cancers and its involvement in tumorigenesis. Clin Cancer Res. 2004;10(24): 8386-8395.

15. Abdel-Fatah TM, Middleton FK, Arora A, et al. Untangling the ATRCHEK1 network for prognostication, prediction and therapeutic target validation in breast cancer. Mol Oncol. 2015;9(3):569-585.

16. Kim MK, Min DJ, Wright G, Goldlust I, Annunziata CM. Loss of compensatory pro-survival and anti-apoptotic modulator, IKKe, sensitizes ovarian cancer cells to CHEK1 loss through an increased level of $\mathrm{p} 21$. Oncotarget. 2014;5(24):12788-12802.

17. Wang J, Huang Q, Liu ZP, et al. NOA: a novel network ontology analysis method. Nucleic Acids Res. 2011;39(13):e87.
18. Raja UM, Gopal G, Rajkumar T. Intragenic DNA methylation concomitant with repression of ATP4B and ATP4A gene expression in gastric cancer is a potential serum biomarker. Asian Pac J Cancer Prev. 2012;13(11):5563-5568.

19. Calvete O, Reyes J, Zuñiga S, et al. Exome sequencing identifies ATP4A gene as responsible of an atypical familial type 1 gastric neuroendocrine tumour. Hum Mol Genet. 2015;24(10):2914-2922.

20. Bruun J, Kolberg M, Ahlquist TC, et al. Regulator of chromosome condensation 2 identifies high-risk patients within both major phenotypes of colorectal cancer. Clin Cancer Res. 2015;21(16):3759-3770.

21. Humphries JD, Byron A, Bass MD, et al. Proteomic analysis of integrinassociated complexes identifies rec2 as a dual regulator of rac1 and arf6. Sci Signal. 2009;2(87):ra51

22. Matsuo M, Nakada C, Tsukamoto Y, et al. Mir-29c is downregulated in gastric carcinomas and regulates cell proliferation by targeting rec2. Mol Cancer. 2013;12:15.

23. Nakamura Y, Tanaka F, Haraguchi N, et al. Clinicopathological and biological significance of mitotic centromere-associated kinesin overexpression in human gastric cancer. Br J Cancer. 2007;97(4):543-549.

24. Shigeishi H, Oue N, Kuniyasu H, et al. Expression of bub1 gene correlates with tumor proliferating activity in human gastric carcinomas. Pathobiology. 2001;69(1):24-29.

25. Grabsch H, Takeno S, Parsons WJ, et al. Overexpression of the mitotic checkpoint genes bub1, bubr1, and bub3 in gastric cancer-association with tumour cell proliferation. J Pathol. 2003;200(1):16-22.

26. Watanabe E, Ohara R, Ishimi Y. Effect of an $\mathrm{mcm} 4$ mutation that causes tumours in mouse on human mcm $4 / 6 / 7$ complex formation. J Biochem. 2012;152(2):191-198

27. Kikuchi J, Kinoshita I, Shimizu Y, et al. Minichromosome maintenance $(\mathrm{mcm})$ protein 4 as a marker for proliferation and its clinical and clinicopathological significance in non-small cell lung cancer. Lung Cancer. 2011;72(2):229-237.

28. Tomita Y, Imai K, Senju S, et al. A novel tumor-associated antigen, cell division cycle 45 -like can induce cytotoxic t-lymphocytes reactive to tumor cells. Cancer Sci. 2011;102(4):697-705.
OncoTargets and Therapy

\section{Publish your work in this journal}

OncoTargets and Therapy is an international, peer-reviewed, open access journal focusing on the pathological basis of all cancers, potential targets for therapy and treatment protocols employed to improve the management of cancer patients. The journal also focuses on the impact of management programs and new therapeutic agents and protocols on

\section{Dovepress}

patient perspectives such as quality of life, adherence and satisfaction. The manuscript management system is completely online and includes a very quick and fair peer-review system, which is all easy to use. Visit http://www.dovepress.com/testimonials.php to read real quotes from published authors. 